\title{
Comments on "Control of a Planar Underactuated Biped on a Complete Walking Cycle"
}

\author{
Bernard Brogliato
}

The above paper [1] possesses several approximations and flaws, which we try to explain. Roughly, the topic concerns the problem of trajectory tracking for a class of mechanical Lagrangian systems subject to unilateral constraints on the generalized position $\phi(q) \geq 0$, $\phi(q) \in \mathbb{R}^{m}$. Such multibody mechanical systems also involve a complementarity relation between the constraint and a Lagrange multiplier $0 \leq \phi(q) \perp \lambda \geq 0{ }^{(1)}$, and generalized velocity jumps (impacts). The complementarity relations and the velocity jump law, form a specific contact model. A contact model is necessary for the chosen model to be meaningful from a mechanical point of view. When dealing with systems of rigid bodies, the complementarity conditions are the simplest way to deal with the contact dynamics: they state that adhesion or magnetic forces are excluded from the model. Such nonsmooth mechanical systems form a special class of complementarity systems, but other formalisms exist [7]. It is worth noting that the complementarity conditions are not included in the model presented in [1], which is therefore incomplete. Specifically, the authors deal with a particular biped robots model that fits within a class of impulsive ODEs, or measure differential equations. We will come back on this later in this note. The tracking problem is examined when the system undergoes an infinity of cycles, each cycle being composed of three phases of motion: single-support phase, double-support phase, and the impact when the feet hit the ground. Apart from possible underactuation, the problem is quite similar to what is tackled in [2]-[5], that concerns fully actuated Lagrangian systems undergoing cycles which consist of free motion phases, constrained motion phases, and transition phases with impacts. The effects of the impacts and of the complementarity relations do not change from one problem to the other one. This is why it is worth understanding the simplest case before tackling more sophisticated control problems (underactuated systems, flexible joint manipulators, to cite a few). It is worth noting that the infinity of cycles (and consequently

Manuscript received June 28, 2005; revised February 7, 2006. Recommended by Associate Editor F. Bullo.

The author is with the INRIA, 38334 Saint Ismier cedex, France (e-mail: Bernard.Brogliato@inrialpes.fr).

Digital Object Identifier 10.1109/TAC.2007.895947

${ }^{1}$ The symbol $\perp$ means that $\phi(q)$ and $\lambda$ have to be orthogonal one to each other. Since they are both non-negative, this is equivalent to the componentwise relation $0 \leq \phi_{i}(q), 0 \leq \lambda_{i}, \phi_{i}(q) \lambda_{i}=0$ for all $1 \leq i \leq m$. 
of impacting and detaching events) is the source of difficulty in this problem.

Globally, there are two main problems with [1].

i) As pointed out in the introduction, the dynamical model neglects fundamentals aspects of the contact dynamics of multibody mechanical systems made of rigid bodies and subject to unilateral constraints $\phi(q) \geq 0$, and this has serious consequences on the tracking control problem.

ii) The stability properties of the closed-loop system (24a) (24b) (24c) as claimed in [1, Prop. 2], are wrong.

The choice of a model is a delicate matter, and we shall come back on point i) later. Let us deal first with item ii). Proposition 2 in [1] states that the origin of the closed-loop system. . . is uniformly Lyapunov stable and all the trajectories converge to zero for almost all $t \geq 0$ and uniformly in the initial conditions. This statement contains three main sub-statements (the third property has to be guessed from the words all the trajectories converge to zero, which I suppose mean for all initial conditions the trajectories converge to zero)

a) the origin of the closed-loop system is Lyapunov stable;

b) the stability is asymptotic;

c) the stability is global.

The last sentence of the proof [1, Prop. 2], i.e. Exponential stability for almost all $t$ follows since the origin of $(24 \mathrm{c})$ is also exponentially stable, makes the reader think that the closed-loop system (24a)-(24c) is exponentially stable, which is stronger than the mere asymptotic stability. For the sake of briefness we will disregard this claim of [1] in this comment and focus on (a)-(c) only. Let us first assume that the dynamical model presented in [1] represents a satisfactory model, and let us show why items (c) and (b) cannot hold. Point (a) will be examined later.

- Point (c): A first flaw in [1, Prop. 2] is that the presented control algorithm in (17), (18), (22a), (22b), and (13) cannot assure the global stability for at least three reasons.

- The reasoning employed in the proof of [1, Prop. 2], supposes that the feedback gains are chosen large enough so that the convergence rate during the "smooth" (impact-free) phases, is fast enough so that the error state goes into some basin of attraction for the next phase. However, this should be related to the initial tracking error.

- Also, the uniformity with respect to the cycles of the feedback gains upperbound which assures the stability, should be proved. Indeed the impacts are seen in [1] as a disturbance that has to be compensated for with high enough gains ${ }^{2}$. One should at least compute the "disturbance" or an upperbound of it, and show that indeed the same gains are suitable at the next cycle. This also seems to be related to the frequency of the walk, as longer periods of impactless motion, permit to augment the decrease of the tracking error (indeed the controllers imply the asymptotic stability of the error when applied independently in each phase, on a infinite time interval). If the result holds independently of the speed of the walk, this should be proved and stated as it seems to be an important feature of the control scheme.

- Third, as explained in [3], the controllers and the systems "modes" (free motion, constrained motion, transition phase for [2]-[4] or single-support phase, double-support phase for [1]) have to be synchronized. This is a basic assumption. It is likely that stability is lost if the biped is initialized in a double-support phase, with the controller designed for the single-support phase. Synchronicity is what allows one to write the closed-loop dynamics as in $[1,(24 a)-(24 c)]$. If the stablity

\footnotetext{
${ }^{2}$ This is not the case in [3], [4] in which passivity-based controllers are used, in order to take advantage of the kinetic energy loss at each impact for the stabilization process.
}

result does not depend on such a synchronicity property then this should be stated as this seems to be an important property.

- Point (b): A second flaw in [1, Prop. 2] comes from of a fundamental reason which prevents the asymptotic stability to hold.

To clarify this point, let us assume that the system is initialized in a single-support phase, with a zero tracking error. Thus until the first impact occurs, the quadratic function $V(\tilde{q}, \dot{\tilde{q}})$ playing the role of a Lyapunov function (defined in [1, eq. (20)]), keeps its value $V(\tilde{q}, \dot{\tilde{q}})=0$. At an impact and according to the standard model of nonsmooth mechanics, the velocity undergoes a jump while the position remains continuous. If the desired trajectory and its first derivative are continuous [which seems to be the case in [1] where the only available information on the desired trajectory design is given in (13)] then a strictly positive jump occurs in the function $V(\cdot)$ at the impact time, and the Lyapunov function becomes positive. This shows that asymptotic stability is impossible to obtain with "asymptotically stable" controllers, except if the jumps in $V(\cdot)$ tend to zero. This is one of the several subtleties of this tracking control problem, solved in [3], [4] by designing the transition-phase desired trajectory in a very specific way. Possibly other ways to deal with this problem can be invented, see for instance, [6] in a quite specific setting, however. To be more precise, the jump in the function $V(\tilde{q}, \dot{\tilde{q}})$ defined in [1, eq. (20)] can be calculated as

$$
\begin{aligned}
V\left(t^{+}\right)-V\left(t^{-}\right) \\
=\frac{1}{2}\left(\dot{\tilde{q}}\left(t^{+}\right)^{T} \dot{\tilde{q}}\left(t^{+}\right)-\dot{\tilde{q}}\left(t^{-}\right)^{T} \dot{\tilde{q}}\left(t^{-}\right)+\epsilon \tilde{q}^{T}\left(\dot{\tilde{q}}\left(t^{+}\right)-\dot{\tilde{q}}\left(t^{-}\right)\right)\right. \\
=\frac{1}{2}\left(\dot{q}\left(t^{+}\right)+\dot{q}\left(t^{-}\right)-\dot{q}_{d}\left(t^{+}\right)-\dot{q}_{d}\left(t^{-}\right)\right)^{T} \\
\\
\quad \times\left(\dot{q}\left(t^{+}\right)-\dot{q}\left(t^{-}\right)-\dot{q}_{d}\left(t^{+}\right)+\dot{q}_{d}\left(t^{-}\right)\right) \\
\\
\quad+\epsilon \tilde{q}^{T}\left(\dot{q}\left(t^{+}\right)-\dot{q}_{d}\left(t^{+}\right)-\dot{q}\left(t^{-}\right)+\dot{q}_{d}\left(t^{-}\right)\right)
\end{aligned}
$$

In order to continue the calculation one needs to choose an impact model which allows one to express $\dot{q}\left(t^{+}\right)$from $\dot{q}\left(t^{-}\right)$and some physical parameters (restitution, unilateral constraints). In [4] Moreau's rule is chosen and the jump $V\left(t^{+}\right)-V\left(t^{-}\right)$is calculated in order to carefully design the desired trajectory during transition phases. Independently of this restitution rule, it however appears from (1) that: 1) the value of $V\left(t^{+}\right)-V\left(t^{-}\right)$depends a lot on the desired trajectory velocity at the impact time, 2) even if perfect tracking occurs before the impact, the jump $V\left(t^{+}\right)-V\left(t^{-}\right)$is not necessarily small, 3) when a nonzero tracking error is present before the impact (which seems to be the most general case), the impact time is not known and this complicates even more the study of the jump in (1), and 4) the fact that the kinetic energy decreases at the impacts, is absent from (1) because the function $V(\tilde{q}, \dot{\tilde{q}})$ does not mimic the systems's kinetic energy. These issues are very vaguely (if not at all) examined in [1]. Point 4) may not be necessary for the design of a stable controller, however one may guess that it should help and that passivity-based controllers certainly are better candidates than feedback linearizing ones in this sort of problems. Clearly some upperbounds of $V\left(t^{+}\right)-V\left(t^{-}\right)$can be calculated as long as the desired trajectory and its velocity are bounded, but this does not contradict point 2) and the fact that asymptotic stability cannot be claimed until a detailed analysis of the variation of $V(\tilde{q}, \dot{\tilde{q}})$ has been done. The claim in the Proof of Proposition 2 in [1] that the perturbation due to the impact may be compensated for, only states an obvious fact: If the function $V(\tilde{q}, \dot{\tilde{q}})$ has undergone a positive jump, then letting the feedback gains being large enough and the impactless motion being long enough after the impacts, one can decrease arbitrarily $V(\tilde{q}, \dot{\tilde{q}})$. However, once again this is not sufficient to prove the asymptotic stability in such infinitely-many-cycles tasks. One has to take great care of the definition of $q_{d}(\cdot)$ in the neighborhood of the impacts, in order to correctly examine (1). The upperbounds in [1, eq. (25), Rem. 1] do not help a lot for the asymptotic stability. This is true even in the one degree of freedom case as studied in [2]. A suitable notion of stability 
has been proposed in [2]-[4] to cope with this issue. It is noteworthy that designing some jump in $\dot{q}_{d}(\cdot)$ to mimic the impact effect, does not straightforwardly solve the problem because the impact times generally are unknown, so that the jump in $\dot{q}(t)$ and $\dot{q}_{d}(t)$ cannot coincide except if perfect tracking occurs.

About the model: The dynamics as it is stated in (24a)-(24c) in [1], can be considered as a measure differential equation (MDE) or as an impulsive ODE of the type studied for instance in [9], i.e., dynamical systems of the form

$$
\begin{cases}\dot{x}(t)=f(x(t)) & \text { if } t \neq t_{k} \\ x\left(t_{k}^{+}\right)-x\left(t_{k}^{-}\right)=g\left(t_{k}, x\left(t_{k}^{-}\right)\right) & \text {if } t=t_{k}\end{cases}
$$

where $x=\left(\tilde{q}^{T}, \dot{\tilde{q}}^{T}\right)^{T}$ in (24a) and (24c). The sequence $\left\{t_{k}\right\}_{k \geq 0}$ may be purely exogenous, or the times $t_{k}$ may be functions of the state $x$, with some restrictions so that they satisfy $t_{k+1}-t_{k} \geq \delta>0$ for some $\delta \in \mathbb{R}$. Such MDEs do not represent a satisfactory model for unilaterally constrained mechanical systems, which are measure differential inclusions (MDIs, see, e.g., [8] and [7] for details on this fundamental point). This is because MDEs do not contain any complementarity relations $0 \leq \phi(q) \perp \lambda \geq 0$, whereas MDIs do. The complementarity conditions are as important as the state jumps for the analysis, the control, the numerical simulation. They should not be considered as a secondary ingredient of the dynamics.

The times and states of detachment from the constraints (when the feet take off due to the control action) also depend on the tracking error and are not known analytically. They can be approximated calculating the solution of a linear complementarity problem (LCP) [4], [7], which is constructed in a classical way from the complementarity relation $0 \leq$ $\phi(q) \perp \lambda \geq 0$ and the second-order Lagrangian dynamics to get $0 \leq$ $\lambda(t) \perp \ddot{\phi}(q(t))=A(q(t)) \lambda+B(q(t), \dot{q}(t), t) \geq 0$ for some semi positive definite matrix $A(q)$ and some vector $B(q, \dot{q}, t)$. No mention of this is made in [1] which focuses on the contacting times (impacts) only and considers them as exogeneous as stated in the first sentence of the proof of Proposition 2. ${ }^{3}$ This is crucial because the controllers have to be switched at the detachment time, and the desired trajectory has to be adapted. If both the controller structure and the desired trajectory do not jump to new values at the accurate detachment time, then the Lyapunov function will undergo some positive jump. At least, one can say that this induces a kind of "disturbance" similar to that produced by the impact. It is by the way a general feature that the complementarity conditions play an as important role as the velocity jumps, though their role is often wrongly neglected. The dynamical coupling between the tangential and normal directions, due to a non block-diagonal inertia matrix, also play a crucial role in the stability analysis (such a coupling clearly appears when a suitable set of generalized coordinates as those in [10] is chosen).

Now that the discrepancy between complementarity systems and impulsive ODEs has been clarified, let us come back to item a) above, on Lyapunov stability. There exists a well-developped and abundant literature that copes with the Lyapunov stability of impulsive ODEs as in (2). Claiming Lyapunov stability results for [1, eq. (24a)-(24c)], should refer to the basic definitions and extensions of Lyapunov's second method for impulsive ODEs (see for instance [8, Def. 7.1, Th. 7.1], [9], and the further references therein). This is not done properly in [1]. Even worse, it is ignored. Assuming that the proposed control strategy allows one to write a closed-loop system as (24a)-(24c) and assuming that this closed-loop system fits within impulsive ODEs as in (2), then it is possible that Lyapunov stability holds, using the quadratic function in (20). But this is not proved in [1]. Here, some

${ }^{3}$ Footnote 3 in [1] points out that a better way to handle impact times would be to include their state dependency. This is a good remark, and this precisely implies to include the complementarity relations in the model. of the arguments contradicting the global and the asymptotic stability can be repeated. In particular one cannot avoid a detailed analysis of (1) to prove that item a) is true. The sentence the perturbation due to the impact may be compensated for to ensure that each DS phase starts off with the right initial condition is not clear to me. What is a right initial condition?

We may conclude that the approach chosen in [1] lies somewhere in-between the complementarity modelling and the impulsive ODE modelling, and that this may be the source of imprecisions and flaws in [1]. Finally, I would like to end this paragraph on the modelling with two comments.

- it is not clear that the proposed controller assures that the closedloop system really fits within (2), despite in [1, eq. (24a)-(24c)] is written as an impulsive ODE. Indeed it is stated at the beginning of the proof of [1, Prop. 2] that the reference trajectories are designed so that the impacts take place at precise instants. I do not see how this can be guaranteed with the proposed controllers if there are nonzero initial tracking errors, since the controllers during the SS and DS phases guarantee the asymptotic convergence of the tracking errors to zero; see [1, Prop. 1 and eq. (23)]. Except if the initial tracking error is zero, there will always be a difference between the planned time of impact, and the real time of impact. The impact and detachment times may be estimated. Even if they could be analytically calculated, they would depend on the initial errors.

- it is a common feature of unilaterally constrained mechanical systems, that they live on subspaces of the configuration space with varying dimensions. Equation (24c) in [1] represents the system in a double-support phase, i.e. with at least two degrees of freedom less than in the single-support phase (corresponding to an additional foot stuck on the ground). This is not visible in (24c), where one would expect to see either a reduced-order dynamical system, or a full-order dynamics with an equality constraint of the form $\phi_{i}(q)=0$ for some $1 \leq i \leq m$, and a Lagrange multiplier. Consider the simplest case of a one degree-of-freedom mass subject to a unilateral constraint: $m \ddot{q}(t)=u(t)+\lambda(t), 0 \leq \lambda(t) \perp$ $q(t) \geq 0$. Suppose that $u(t)=m \ddot{q}_{d}(t)-\gamma_{1} \dot{\tilde{q}}(t)-\gamma_{2} \tilde{q}(t)$. The closed-loop dynamics is $m \ddot{\tilde{q}}(t)+\gamma_{1} \dot{\tilde{q}}(t)+\gamma_{2} \tilde{q}(t)=\lambda(t)$, $0 \leq \lambda(t) \perp q(t) \geq 0$. Suppose that on some strictly positive time-interval $I$ one has $q(t)=0$. The closed-loop dynamics is $m \ddot{q}_{d}(t)+\gamma_{1} \dot{q}_{d}(t)+\gamma_{2} q_{d}(t)=-\lambda(t), q(t)=0$ on $I, 0 \leq$ $\lambda(t) \perp q(t) \geq 0$. This is an algebraic equation relating the contact force and the external action (the control). The constraint remains active on $I$ if and only if $\lambda(t)>0$ on $I$. Detachment occurs at $t \in I$ if $\lambda(t)=0$ and $\ddot{q}(t)>0$. At all $t \in I$, the multiplier $\lambda(t)$ is computed as the unique solution of the LCP $0 \leq \lambda(t) \perp \ddot{q}(t)=(1 / m) u(t)+(1 / m) \lambda(t) \geq 0$. Therefore, detachment or sticking depends on $q_{d}(\cdot)$ and its derivatives. Moreover let us choose a simple quadratic function $V(\tilde{q}, \dot{\tilde{q}})$ that is a Lyapunov function for the unconstrained case, playing a role similar to (20) in [1]. How does this function vary in $I$ and along the infinite switches between constrained and free motion phases? One cannot disregard the value of $q_{d}(\cdot)$ on $I$ to lead a stability analysis. To say nothing on the analysis at the impact times. Control strategies and adapted stability notions that cope with such issues are the object of [2]-[4].

Conclusions: To recapitulate on the mistakes and the imprecisions of [1]:

- given in [1, eq. (24a)-(24c)], points b) and c) above are wrong, point a) is perhaps true but is not proved,

- the closed-loop system [1, eq. (24a)-(24c)] does not result from the proposed controllers,

- the model is incomplete as some contact conditions are missing. 
It is possible that the control strategy that is proposed in [1], and which is based solely on the rate of decrease of $V(\tilde{q}, \dot{\tilde{q}})$ during impactless motion, may yield some kind of ultimate boundedness or practical stability of the state on $[0,+\infty$ ) (in fact the strategy of [1] is quite close to what is examined in $[2, \S \mathrm{V} . \mathrm{B}]$, in which it was already pointed out that except in very special cases, feedback linearization may not be the right method to apply due to the difficulty to estimate the jump in the Lyapunov function at impact times). However, this result is not proved in [1] which rather tackles global asymptotic uniform stability. Especially, a detailed computation of (1) at impact times, and the presentation of how detachment is managed, are mandatory before any stability result can be claimed. The problem that is tackled in [1] can therefore be considered as being still open. One problem the authors could start with is: what happens if the control strategy is applied to a one degree of freedom system (a controlled mass with a complementarity condition and a simple Newton's restitution law)? Then, proceed to the fully actuated $n$-degree-of-freedom case with any inertia matrix (notice that the dynamical coupling between normal and tangential directions also increases the difficulty of the analysis). Then, reconsider the biped control problem and tackle another type of stability.

\section{REFERENCES}

[1] A. Chemori and A. Loria, "Control of a planar undercatuated biped on a complete walking cycle," IEEE Trans. Autom. Control, vol. 49, no. 5, pp. 838-843, May 2004.

[2] B. Brogliato, S. I. Niculescu, and P. Orhant, "On the control of finite-dimensional mechanical systems with unilateral constraints," IEEE Trans. Autom. Control, vol. 42, no. 2, pp. 200-216, Feb. 1997.

[3] B. Brogliato, S. I. Niculescu, and M. D. P. Monteiro-Marques, "On tracking control of a class of complementary-slackness hybrid mechanical systems," Syst. Control Lett., vol. 39, pp. 255-266, 2000.

[4] J. M. Bourgeot and B. Brogliato, "Tracking control of complementarity Lagrangian systems," Int. J. Bifurc. Chaos, vol. 15, no. 6, pp. 1839-1866, Jun. 2005.

[5] J. M. Bourgeot, "Contribution à la commande de systèmes mécaniques non-réguliers," Ph.D. dissertation, Inst. National Polytech. Grenoble, Grenoble, France, Oct. 2004

[6] L. Menini and A. Tornambe, "Asymptotic tracking of periodic trajectories for a simple mechanical subject to nonsmooth impacts," IEEE Trans. Autom. Control, vol. 46, no. 7, pp. 1122-1126, Jul. 2001.

[7] B. Brogliato, "Some perspectives on the analysis and control of complementarity systems," IEEE Trans. Autom. Control, vol. 48, no. 6, pp. 918-935, Jun. 2003.

[8] B. Brogliato, Nonsmooth Mechanics, ser. CCE. London, U.K.: Springer-Verlag, 1999.

[9] D. D. Bainov and P. S. Simeonov, Systems With Impulse Effects: Stability, Theory and Applications, ser. Ellis Horwood Series in Mathematics and its Applications. New York: Wiley, 1989.

[10] N. H. McClamroch and D. Wang, "Feedback stabilization and tracking of constrained robots," IEEE Trans. Autom. Control, vol. 33, no. 5, pp. 419-426, May 1988. 